\title{
Universal kinetics for engagement of mechanosensing pathways in cell adhesion
}

\begin{abstract}
When plated onto substrates, cell morphology and even stem cell differentiation are influenced by the stiffness of their environment. Stiffer substrates give strongly spread (eventually polarized) cells with strong focal adhesions, and stress fibers; very soft substrates give a less developed cytoskeleton, and much lower cell spreading. The kinetics of this process of cell spreading is studied extensively, and important universal relationships are established on how the cell area grows with time. Here we study the population dynamics of spreading cells, investigating the characteristic processes involved in cell response to the substrate. We show that unlike the individual cell morphology, this population dynamics does not depend on the substrate stiffness. Instead, a strong activation temperature dependence is observed. Different cell lines on different substrates all have long-time statistics controlled by the thermal activation over a single energy barrier $\Delta G \approx 19 \mathrm{kcal} / \mathrm{mol}$, while the early-time kinetics follows a power law $\sim t^{5}$. This implies that the rate of spreading depends on an internal process of adhesion-mechanosensing complex assembly and activation: the operational complex must have 5 component proteins, and the last process in the sequence (which we believe is the activation of focal adhesion kinase) is controlled by the binding energy $\Delta G$.
\end{abstract}

Insert Received for publication Date and in final form Date.

\section{Introduction}

Matrix stiffness is known to affect cell size and morphology $(1,2)$. When cells are plated onto soft substrates, their footprint will not increase as much as on stiff substrates, and their spreading will be more isotropic: resulting cells will be round and dome-like in shape. On stiff substrates, the same cells will spread very strongly, develop concentrated focal adhesion clusters and stress fibers of bundled F-actin, and eventually polarize to initiate migration. This leads to several well-documented biological functions in tissues: variable stem-cell differentiation pathways $(1,3)$, the fibroblastmyofibroblast transition near scar tissue (4-6), fibrosis in smooth-muscle cells near rigid plaque or scar tissue $(7,8)$, and the stiffer nature of tumor cells $(9,10)$. The definitive review (11) summarizes this topic. There are several different mechanosensing processes acting either simultaneously, or in different circumstances. One of the key physical mechanisms of stiffness sensing is thought to be based on the extracellular latent TGF- $\beta$ complex $(5,12)$; another key mechanism involves the intracellular complex incorporating integrin and focal adhesion kinase (FAK) (13-15). There is also a lot of discussion of integrin itself, or vinculin, using their catch-bond characteristics to produce mechanosensing $(16,17)$, however, we would argue that these proteins do not possess a catalytic domain and therefore cannot produce a required chemical signal: the output of a mechanosensor (15). It is important to note that many catch-bond models

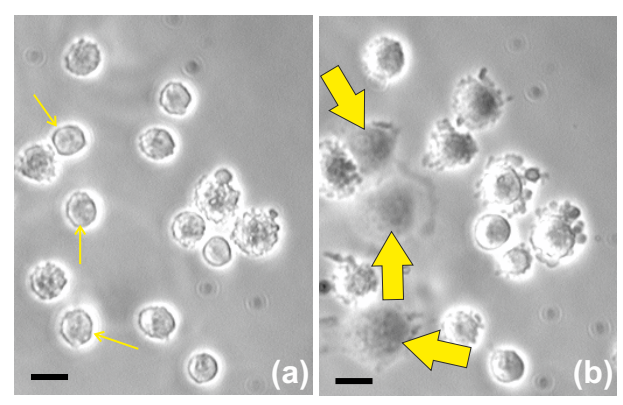

Figure 1: Illustration of mechanosensing response and cell spreading. Plots (a) and (b) show the same cells: immediately after planting on the substrate (solid glass with fibronectin), and 15 min later, when several cells have already responded by spreading (labeled by mathcing arrows). Scale bar $=20$ $\mu \mathrm{m}$.

use the collective activity of many integrins in mature focal adhesion clusters, while in this work we focus on the properties of individual adhesion-mechanosensing protein complexes. This is more important in the initiation of spreading, where mature clusters are yet to develop. We also emphasize that here, and in the rest of this paper, we are discussing isolated cells on a substrate: when cells adhere to each other, their shape transitions are controlled by other mechanisms, based on cadherin and associated pathways (18). 
bioRxiv preprint doi: https://doi.org/10.1101/292367; this version posted April 4, 2018. The copyright holder for this preprint (which was not certified by peer review) is the author/funder. All rights reserved. No reuse allowed without permission.

The dynamics of cells spreading has been studied extensively, and several characterstic universal features have been established $(11,19,20)$. In particular, the average cell area has been shown to grow with time as a power law, often with the radius of cell footprint being $R \propto t^{1 / 2}(21-23)$. Several mechanistic models of how the cell spreading is achieved after the adhesion to extracellular matrix (ECM) is established $(19,21,23)$. However, these papers deal with the characteristic rate of spreading for individual cells, and so neglect stochastic effects in the population dynamics. Here, we observe population dynamics directly, and account for variability in the spreading behavior over different cells.

While reporting and discussing the cell area increase on stiffer substrates, the seminal paper (2) also presents some data on the time-dependence of cell spreading, which already gives a hint for our central experimental finding: the time for cells to engage their mechanosensing pathways, and spread, does not depend on the substrate. In this paper we investigate the time-dependence (kinetics) of mechanosensing response, asking the question: how long does it take for the cell to recognize the nature of its substrate, and respond by engaging the signaling pathways and enacting the required morphological change (spreading on the substrate)? Figure 1 illustrates the point: plots (a) and (b) show the same cells: immediately after planting on the substrate, and after some time, when several cells have already responded by engaging their spreading. We plated two very different cell lines (NIH/3T3 fibroblasts and EA.hy927 endothelial cells) on a variety of substrates that span the range of stiffness from $30 \mathrm{GPa}$ (stiff glass) to $460 \mathrm{~Pa}$ (very soft gel), registering the characteristic time at which the initially deposited planktonic cells engage their mechanosensing response.

We discover three remarkable and unexpected facts: [1] the rate of mechanosensing response is completely universal, not depending on the stiffness of substrates (in contrast to the final cell morphology, which strongly depends on it); [2] the rate-limiting process in mechanosensing response is the same in all cells, and is the activation of FAK (with the energy barrier $\Delta G \approx 19 \mathrm{kcal} / \mathrm{mol}$ in good agreement with simulation studies); [3] the onset of mechanosensing response is controlled by the kinetics of the adhesion-sensor complex assembly, its universal power-law dependence $t^{5}$ suggests that there are exactly four steps in the complex assembly, i.e. five constituting proteins (followed by the FAK activation step of sensor firing). We also measure the sum of the binding energies of proteins in this assembly process, and find that this is non-universal: it depends on the cell type and probably affected by their biological function.

\section{Materials and Methods}

\section{Cells and cell culture procedures}

We chose to study endothelial cells and fibroblasts because their adhesion behaviour is important for understanding cardiovascular diseases and tissue engineering. There are different types of endothelial and fibroblast cells available. Primary cells are directly taken from donor tissue and then grown in cell culture conditions. They can be grown in culture for a specific amount of time before they undergo senescence and die. The advantage of primary cells is that they are as close to in-vivo cells as possible, but as they are taken from different donors, their behaviour is less reproducible. Immortalized cell lines are obtained from primary cells by, for example, transfection or fusion. This results in a change in their DNA, leading to indefinite proliferation. This makes it easier to handle them in multiple long-term experiments, and makes such experiments more reproducible, but at the same time many immortalized cells have some tumorous behavior $(24,25)$. We choose to use immortalized cell lines: NIH/3T3 murine fibroblasts (obtained from ATCC) and EA.hy927 endothelial cells.

NIH/3T3 fibroblasts are very well characterized, as they have been used in many cell studies since their establishment as cell line; they have also been used in cell adhesion studies, making them a good choice for our experiments $(26,27)$. EA.hy 927 is a cell line established in 1983 by the fusion of HUVEC with a lung carcinoma line (28). It has since become a widely used and thus well characterized cell line, popular in studies of cardiovascular diseases. EA.hy927 have also been used for adhesion strength assays (29).

Cells were normally cultured at $37 \mathrm{C}$ and $5 \% \mathrm{CO} 2$ in Dulbecco's modified Eagle's medium (Greiner) with $10 \%$ fetal bovine serum and 1\% Pen/Strep (solution stabilized, with 10,000 units penicillin and $10 \mathrm{mg}$ streptomycin/mL), from Sigma Aldrich (this standard medium is abbreviated as DMEM). For a comparative study of the role of nutrient in the medium, we also used phosphate-buffered saline (PBS), from Thermo Fisher Scientific during the spreading experiments. Cells were subcultured in DMEM every 3 days, at about $70 \%$ confluency, by trypsinization, to avoid the formation of big lumps of cells, thus ensuring that we maintain a single cell suspension. Cells were trypsinized for $5 \mathrm{~min}$ (Trypsin-EDTA $0.05 \%$ ). The solution was then neutralized by added complete growth medium and centrifuged at 1000 rpm for $5 \mathrm{~min}$.

The use of Pen-Strep can be questioned. Antibiotics have been used prophylactically to prevent bacterial infections in cell culture for many years, and they are still being used. It was the introduction of antibiotics that allowed the widespread development of cell culture methods in the first place, as bacterial contamination was a major problem (30). However, although toxicity experiments found that antibiotics were harmless to mammalian cells (31), there are concerns about the use of antibiotics in cell culture associated

Biophysical Journal 00(00) 1-10 
with a neglect of aseptic technique and possible side effects of antibiotics. Many adhesion strength studies use Pen/Strep or other antimycotic or antibiotic solutions in the cell culture, and we followed this procedure as well. We have tested our results on several parallel cell cultures that did not use Pen/Strep, and confirmed that no significant difference was inflicted on our results.

\section{Substrates of varying stiffness}

To span a wide range of substrate stiffness, we used standard laboratory glass (elastic modulus $30 \mathrm{GPa}$ ), and several versions of siloxane elastomers: Sylgard 184 and Sylgard 527 , the latter used with the compound/hardener ratio of 1:1 and 5:4. The resulting elastomers were tested on a standard laboratory rheometer (Anton Paar), giving the values of equilibrium modulus $G=460 \mathrm{~Pa}$ for (S527 5:4), $480 \mathrm{kPa}$ (for S184), and $30 \mathrm{GPa}$ for glass (zero-frequency limit shown in the Supplementary plot, Fig. S1). For comparison, the stiffness of typical mammalian tissues is commonly reported as: $100 \mathrm{~Pa}-1 \mathrm{kPa}$ in brain tissue; $\sim 3 \mathrm{kPa}$ in adipose tissue; $10-20 \mathrm{kPa}$ in muscle; $30-50 \mathrm{kPa}$ in fibrose tissue; up to a few MPa for bone. We avoided applying the commonly used plasma treatment, as this was making the surface highly uneven on a micron scale, which would affect the adhesion. All surfaces were cleaned by ultrasonication in $96 \%$ ethanol for $15 \mathrm{~min}$, and then incubated with $10 \mu \mathrm{g} / \mathrm{mL}$ fibronectin in PBS for $45 \mathrm{~min}$.

\section{Experimental procedure and data acquisition}

In our standard cell-spreading experiment, the cell culture dish was inserted into a closed chamber that maintained controlled temperature with an active water bath, and the $\mathrm{CO}_{2}$ atmosphere, while allowing a microscope observation from the top. The cell culture (density $5 \times 10^{5}$ cells per $\mathrm{ml}$, counted by the Neubauer chamber) was placed over the entire substrate. Cells were left to adhere to the substrate for $2 \mathrm{~min}$, at which point the culture dish containing the substrate is filled slowly with fresh medium to reduce the cell density. This was to prevent new cells depositing, and cell clusters forming on the substrate. Only the cells attached to the substrate at this point were included into the subsequent counting.

To obtain a population distribution of the onset time of cell spreading, we had to choose a spreading criterion, which would be clear and easily distinguishable to avoid counting errors. We choose to count the initial onset of visible spreading, seen as the transition between the near-spherical cell initially planted (physically attached) on the substrate, and the cell with adhesion processes engaged and its shape developing an inflection zone around the rim (see Supplementary Fig. S2 for illustration). This turns out to be easily identified as the near-spherical cell has the sharp edge, and also a lensing effect of focusing light, which disappears on the transition to a more flattened shape. A schematic of this can be seen in Fig. S3. It must be emphasized, that in order for our cell counting to be meaningful, the cells have to be isolated on the substrate: once the cells come into contact with each other, many other mechanosensing mechanisms engage (based on cadherins, and other cell-adhesion systems), and they spread much more readily and more significantly. That is why our initial cell density was chosen such that the initial attachment is in isolation, and our spreading criterion is applied before they spread sufficiently to come in contact (as some cells in Fig. 1 have done).

In each individual experiment (given substrate, fixed temperature, and other parameters), once the cells were deposited on the substrate, and the clock started, we took broad-field microscopic images at regular time intervals, and counted the fraction of cells that have crossed the threshold defined by our spreading criterion. This produced a characteristic sigmoidal curve for each experiment (see Fig. 2): the fraction of cells 'engaged in spreading' starting from zero at $t=0$ and saturating at near- $100 \%$ at very long time (if we exclude the occasional cell mortality). The typical sample size was 100-120 cells in each experiment (field of view). The main sources of error were: inconsistency of application of the spreading criterion in image analysis, imperfections of fibronectin coverage on substrate, temperature fluctuations, and of course the natural cell variability. All of these are random errors, with no systematic drift. We were satisfied that the results were reproducible, and errors did not dominate the data trends.

\section{Results}

It is well-established that cells spread differently on substrates of differing stiffness (there is also a strong dependence on the ECM protein coverage (32), but this was not a variable in our study). The area of a spread cell is invariably greater on stiff substrates, and the shape has different features: more round and symmetric on soft substrates and highly asymmetric, with large strong focal adhesions on stiff substrates (the key article by Janmey et al. (2) makes a very detailed study of this effect). We also see the same effect, for both our cell types, on all substrates.

The first, unexpected, result is presented in Figure 2. These two plots illustrate the cumulative population dynamics: after planting, cells spend several minutes making a decision before starting their spreading - to a wide-area footprint on stiff substrates, or to a more round dome-shape on soft substrates, in agreement with classical studies (1-3) - while the timing of cell spreading is completely insensitive to the substrate nature. The kinetics of mechanosensing response is exactly the same on each substrate; the point of steepest gradient in the cumulative curves in Fig. 2 marks the most probable time for the onset of cell spreading. The work of Sheetz et al. (33) has reported a similar effect (the rate of 
bioRxiv preprint doi: https://doi.org/10.1101/292367; this version posted April 4, 2018. The copyright holder for this preprint (which was not certified by peer review) is the author/funder. All rights reserved. No reuse allowed without permission.
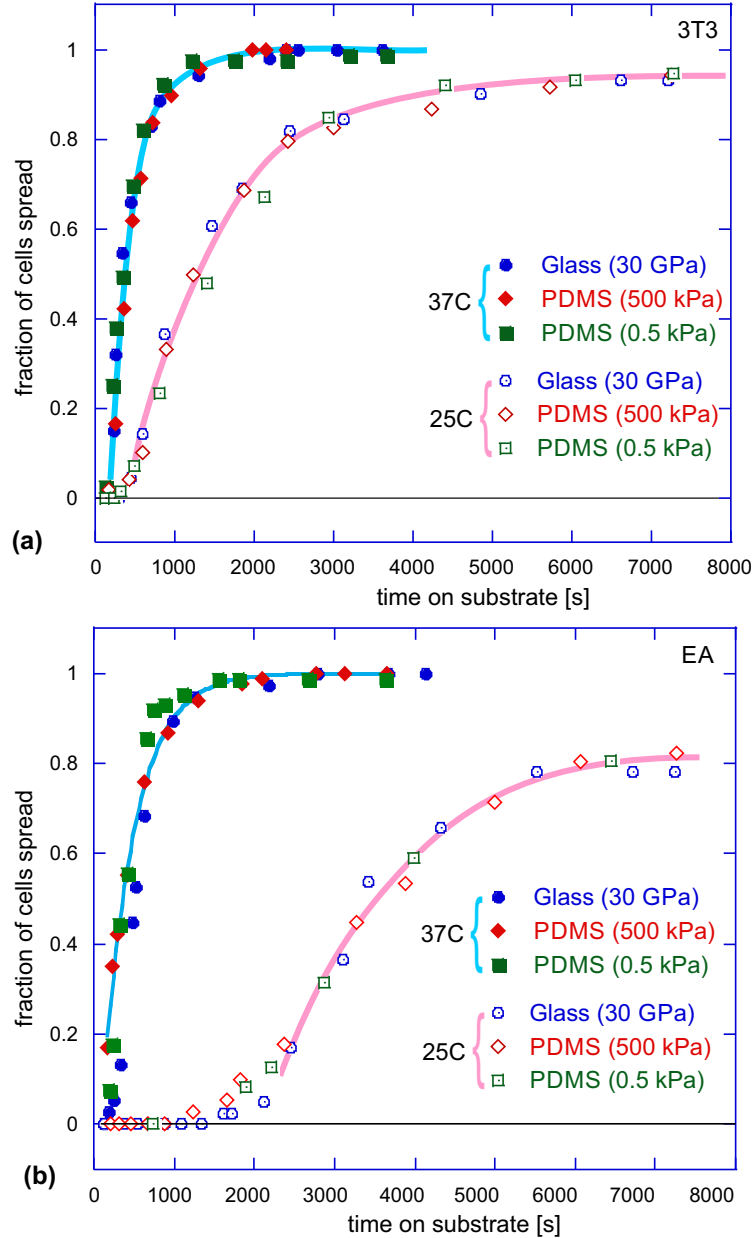

Figure 2: Cumulative population dynamics of cell spreading. Plots (a) and (b) show the growing fraction of cells engaged in spreading on substrates with different stiffness for 3T3 fibroblasts and EA endothelial cells at two different temperatures each. It is clear that the dynamics is not affected by the substrate stiffness, but changes with temperature. In the remainder of this paper, we analyze in detail the long-time behavior of these cumulative curves as they approach saturation, and the behavior at short times when the onset of mechanosensing response occurs.

spreading did not depend on the degree of ECM protein coverage on the surface). The second remarkable fact revealed in Fig. 2 is that the kinetics of cell spreading is strongly affected by temperature.

This leads us to the main conclusion of this paper: the rate of mechanosensing response is an internal cell characteristic, determined by the nature of its sensor and the signaling pathways that translate the sensor output into the morphological response of the cell. The magnitude of this response is affected by the sensor signal strength - but the timing of this process is determined by the internal cell organization, and its thermally-activated processes.
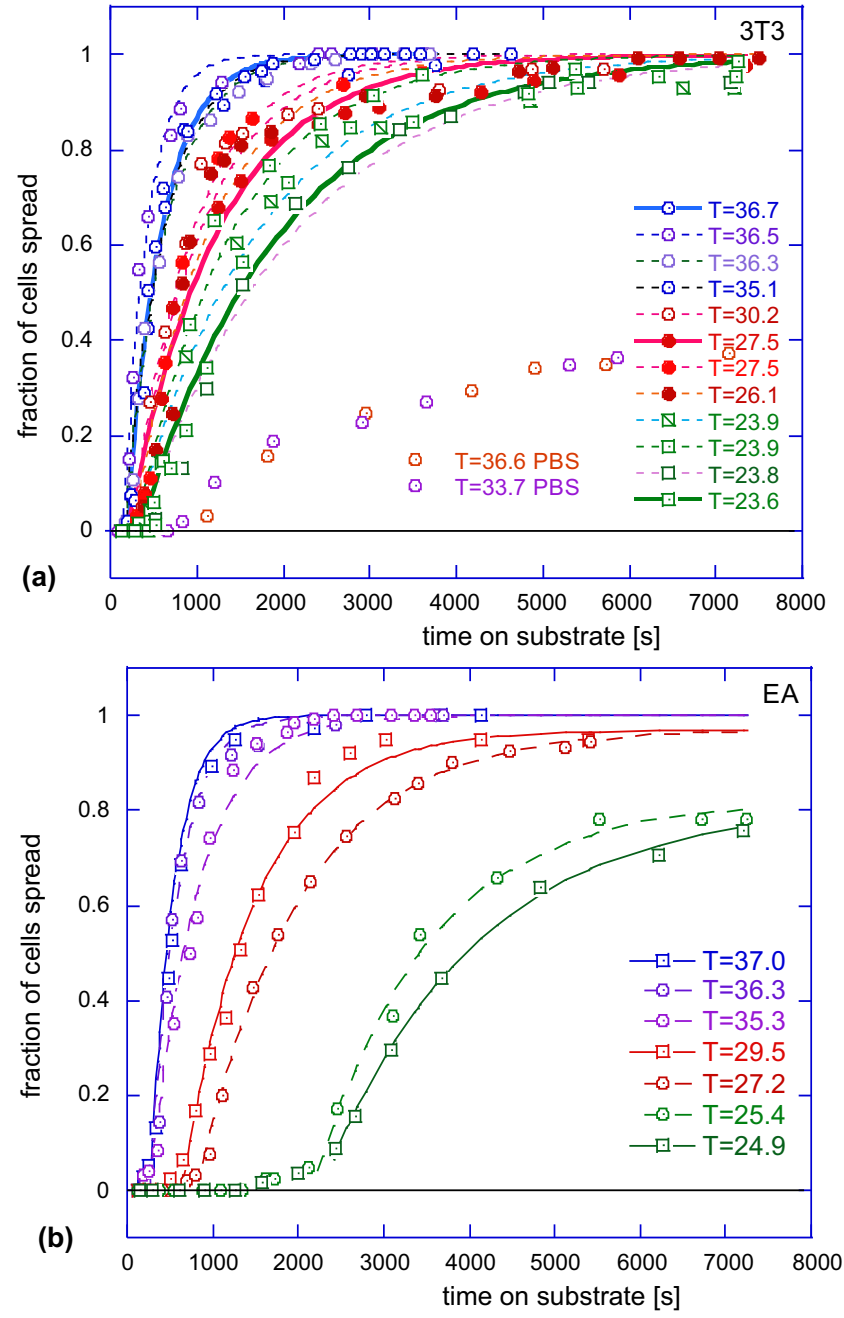

Figure 3: Cumulative population dynamics of cell spreading. Plots (a) and (b) show fraction of spreading cells on glass, at many different temperatures; for $3 \mathrm{~T} 3$ fibroblasts and EA endothelial cells. Lines in all plots are the fits of the longtime portion of data with the exponential relaxation curves, producing the fitted values of the longest relaxation time $\tau$ (see text).

\section{Long-time trend: rate limit of mechanosensing}

To examine the effect of temperature in greater detail, in Fig. $3(a, b)$ we plotted the same cumulative spreadingdynamics curves for the two cell types on glass (as we are now assured that these curves are the same on all substrates). It is noticeable that the initial lag is greater in the EA cells, and that at low temperature the saturation level drops significantly below $100 \%$ - presumably because more cells disengage (or die) at low temperature, reducing the saturation fraction. The same effect is much enhanced for the the nutrient-starved cells in the PBS medium, see in Fig. 3(a): the kinetics of mechanosensing response is very slow in this

Biophysical Journal 00(00) 1-10 


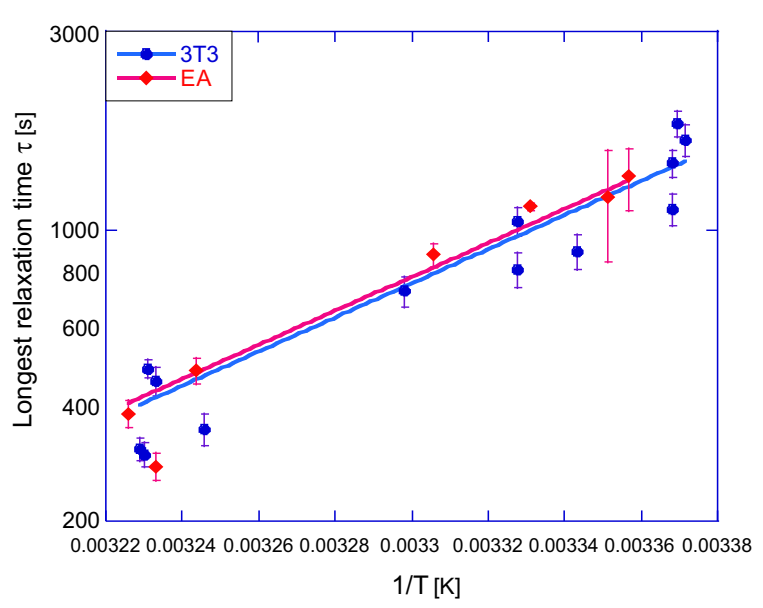

Figure 4: The Arrhenius plot of the longest relaxation time $(\log (\tau)$ vs. inverse absolute temperature) from the exponential fits in Fig. 3 (a,b), giving the same value of binding energy $\Delta G \approx 19 \mathrm{kcal} / \mathrm{mol}$, for both types of cells.

case, and a large fraction of cells do not engage at all, which is not surprising because the ATP-consuming processes are involved in generating the cytoskeletal force and in converting Rho GTPases. But the generic shape of the cumulative curve is universal, and the random spread of data within each individual experiment is not excessive. We then look to analyze the trends in this time dependence.

The curves of the generic shape seen in Figs. 2 and 3 are encountered in many areas of science, and their characteristic foot at early times, especially obvious at lower temperatures, is usually associated with a lag in the corresponding process. We will discuss this early-time regime separately, later in the paper, but first we fit exponential relaxation curves to the long-time portion of the data (as the fit lines in Fig. 3 indicate): $Q(t)=A \cdot\left(1-\exp \left[-\left(t-t_{\text {lag }}\right) / \tau\right]\right.$. The Supplementary Information gives the table of values of $A$ and $\tau$ for each curve, but it is clear from the plots that the fitting to the single-exponential relaxation law, with just two parameters since $A$ is known for each curve, is very successful. The characteristic relaxation time $\tau$ markedly increases at low temperatures. It is interesting that such a characteristic time associated with the 'spreading of an average cell' has been discussed in (19), giving the same order of magnitude (of the order of magnitude 50-100s).

To better understand this dependence on temperature, we tested a hypothesis that this relaxation time is determined by the thermally-activated law by producing the characteristic Arrhenius plots of relaxation times, for both cell types, see Figure 4. It is remarkable that both cells show almost exactly the same trend of their relaxation time: the rate limiting process in their mechanosensing pathways is the same: $\tau=\tau_{0} e^{\Delta G / k_{B} T}$, with the activation energy $\Delta G \approx 18.7 \pm 1.5 \mathrm{kcal} / \mathrm{mol}$, and the thermal rate of attempts $\tau_{0}^{-1} \approx 4 \times 10^{10} \mathrm{~s}^{-1}$. Both values are very sensible: this magnitude of $\Delta G$ is typical for the non-covalent bonding energy between protein domains (34), and this rate of thermal collisions is in excellent agreement with the basic Brownian motion values.

\section{Early-time trend: rate of complex assembly}

After discovering that the late-times (rate-limiting) dynamics of mechanosensing response is quite universal, across different cells and substrates, it becomes clear that the marked difference between different curves in Fig. 3 lies in the earlytime behavior: something that we have called a 'lag' following many similar situations in protein self-assembly. To examine this early-time regime more carefully, we re-plotted the same time series data on the log-log scale in Fig. 5 (a,b).

First of all, from examining these plots it is clear that there is no such thing as a lag time: the log-log plotting reveals that the process is active from the very beginning $(t=0)$ and the plotted value grows as a power-law of time. The only reason that we appear to see a 'lag' is because our experimental technique of counting the cells engaging in spreading did not permit values below $0.01(1 \%)$ to be resolved in this plot; the same certainly applies to other experimental situations reporting similar kinetic data. The trend illustrated in Fig. 5 is clear: the early onset of cell spreading follows the universal power law, and the fitting of all our data sets gives $Q(t)=\alpha t^{5}$ with very good accuracy, where only the prefactor $\alpha$ depends on temperature and the cell type. We find this result truly remarkable: similarly to the universal value of binding energy that controls thermallyactivated rate-limiting relaxation time $\tau$, this very specific $t^{5}$ power law appears to be the only sensible fit of the early-time data for different cells, temperatures, and substrates.

However, the strong temperature dependence is evident in the short-time onset of mechanosensing: the difference was evident in Figs 2 and 3, but is very clearly enhanced in Fig. 5. What changes between the data sets is the prefactor $\alpha$ of the universal power law $\alpha t^{5}$, which has a systematic temperature dependence (the fitted values of $\alpha(T)$ are listed in the Supplementary). Now expecting the thermally activated behavior, by analogy with the earlier analysis, we plot these prefactors $\alpha(T)$ on the Arrhenius plot in Fig. 6. The fitting to $\alpha=$ const $\cdot e^{-\Delta H / k_{B} T}$ indeed gives a very reasonable trend, with the activation energies $\Delta H=70 \mathrm{kcal} / \mathrm{mol}$ for $3 \mathrm{~T} 3$, and $129 \mathrm{kcal} / \mathrm{mol}$ for EA. Note that, in contrast to Fig. 4 , here we have a negative exponent, i.e. the parameter $\alpha(T)$ represents a reaction rate rather than a relaxation time. In the classical Arrhenius-Kramers thermal activation, the process time is shorter as the temperature increases, while the Fig. 6 shows the scaling factor $\alpha(T)$ is decreasing as the temperature decreases instead (which is reflected in the overall observation of longer lag time in the cumulative curves). The magnitudes, and the difference in the energy barriers between the 

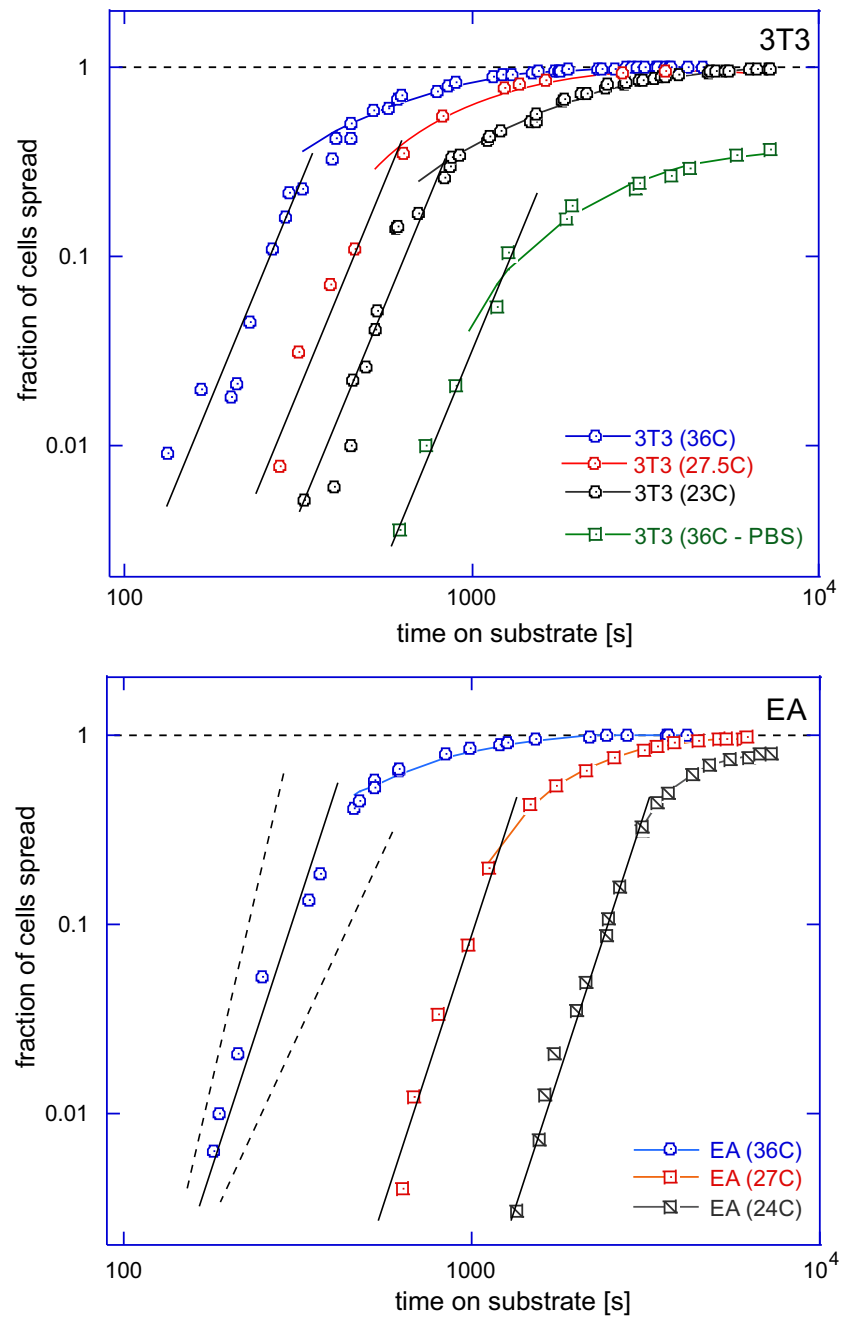

Figure 5: Analysis of the short-time dynamics of cell spreading. Plots (a) and (b) show selected data sets from the Fig. reffig:cumuls2 (a,b), presented on the log-log scale to enhance the short-time dynamical range. In both plots, the power-law slopes of the short-time data follow the equation: $\alpha t^{5}$, with the coefficient prefactor $\alpha$ depending both on cell type and on temperature. The dashed line illustrate the slopes of $t^{6}$ and $t^{4}$ to illustrate the strength of fit.

two cell types make sense because, due to their biological function, the mechanosensing process in fibroblasts should start faster. However, we have so many different quantitative facts and trends that it is necessary to look much more carefully at what we understand about mechanosensing.

\section{Discussion}

To have such a mechanosensor operational, the cell needs to assemble the protein complex connecting and transmitting the pulling force from the F-actin terminals in the cytosol to the extracellular matrix (ECM). The mechanosensor has

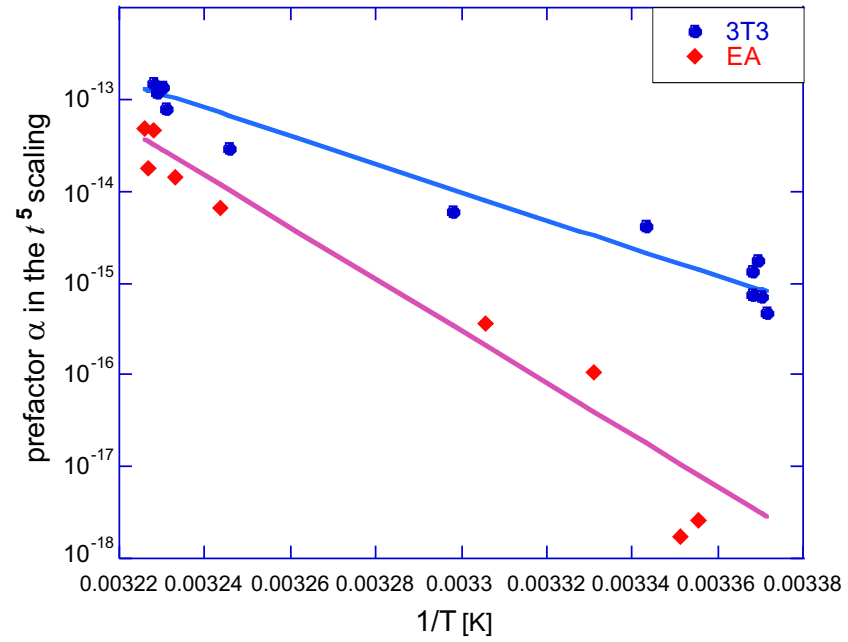

Figure 6: Analysis of the short-time dynamics of cell spreading. The Arrhenius plot of the prefactor $\alpha(T)$, with the fit lines giving the effective activation enthalpy $\Delta H \approx$ $70 \mathrm{kcal} / \mathrm{mol}$ for $3 \mathrm{~T} 3$, and $129 \mathrm{kcal} / \mathrm{mol}$ for EA. See text, explaining how this value represents the sum of free energy barriers of key proteins assembling into the adhesionmechanosensing complex.

to transform these mechanical forces into a chemical signal, which is then starting one or several signaling pathways in the cell that lead to a morphological response (35-37): usually the enhancement of cytoskeleton by increasing the number and branching of F-actin, and activating more myosin motors. It is relatively well accepted that FAK is the central player in this signaling network $(14,35,38,39)$. A recent model of FAK as a mechanosensor (15) shows how the rate of its activation is sensitive to the stiffness of substrate, and the cytoskeletal pulling force, see some details in the Supplementary Information. Importantly, when the force is low (as we would expect at early times, before the mechanosensing pathways are activated and the cytoskeletal forces increase), this rate is controlled only by the bonding energy between its FERM and kinase domains, not the stiffness. A recent molecular-dynamics simulation (34) has explicitly calculated this bonding energy as $\Delta G \approx 17 \mathrm{kcal} / \mathrm{mol}$. If we associate this barrier with the longest relaxation time examined in Fig. 4, the agreement of the $\Delta G$ values is remarkably close. It makes sense that the mechanosensor itself should be the rate-limiting step of any signaling pathway!

What is the physical process underlying the apparently universal power law $\alpha t^{5}$ at the early stages of mechanosensing activation? Although previous work on cell spreading has also talked about power law behavior $(19,21,23)$, it is important not to confuse the role of viscous dissipation in a spreading cell with the overall stochastic population dynamics of the onset of spreading. We believe the origin of this universal time dependence is the dynamic assembly of

Biophysical Journal 00(00) 1-10 


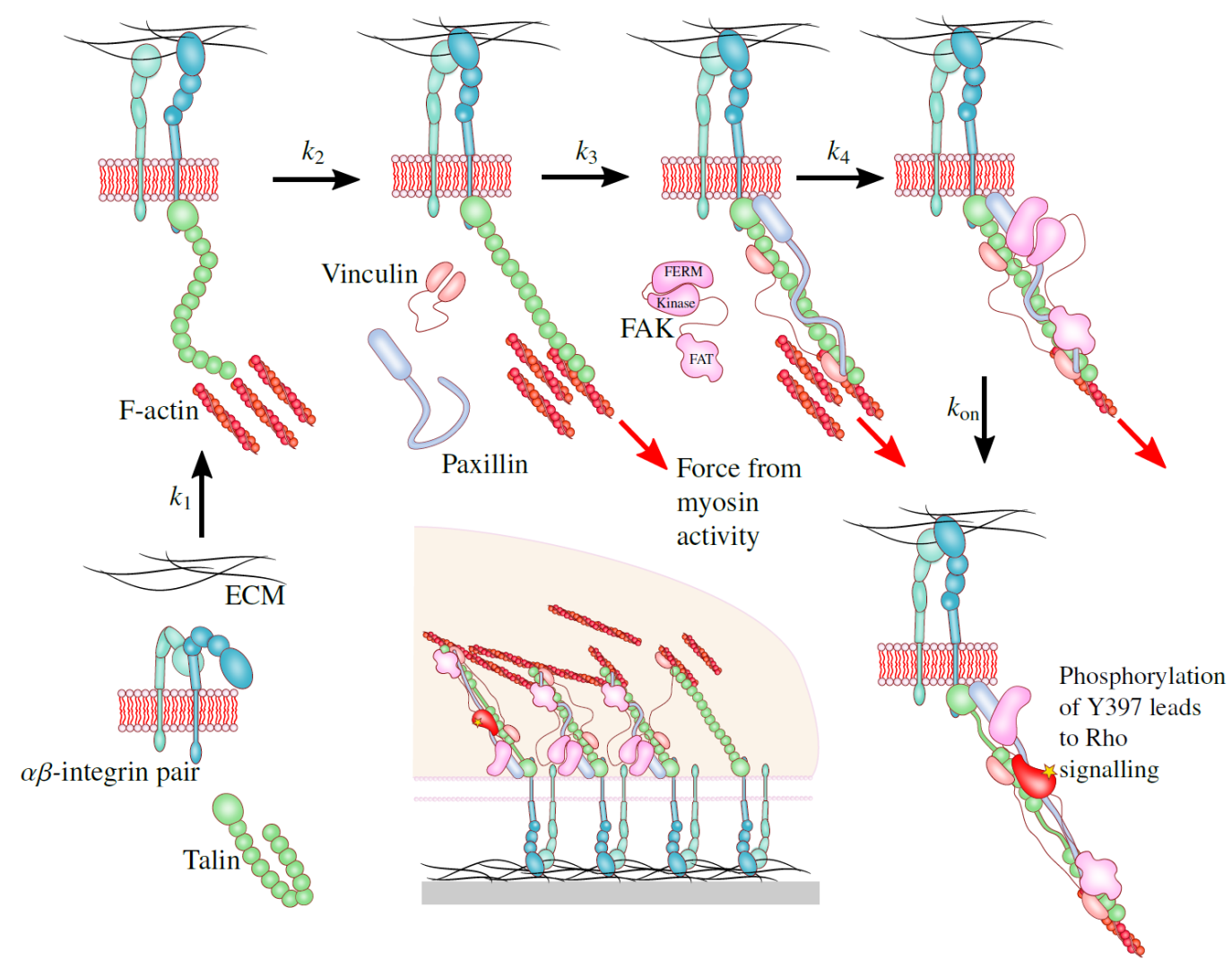

Figure 7: Assembly of a mechanosensor complex. Our analysis suggests that there are five distinct slow stages illustrated in the sequence, with their respective rates $k_{1}-k_{4}$ and the rate of FAK activation $k_{\text {on }}$ (controlled by the free energy barrier $\Delta G \approx 19 \mathrm{kcal} / \mathrm{mol}$, cf. Fig. 4). The product of the five rate constants $\alpha=k_{1} k_{2} k_{3} k_{4} k_{\text {on }}$ is what we measure in the Arrhenius plot Fig. 6. In the center is a sketch of forming focal adhesion cluster, where the individual mechanosensor complexes in various stages of development/turnover are bound by vinculin and actin crosslinking (40).

the integrin-FAK protein complex that is required to transmit the cytoskeletal pulling force across the cell membrane to the ECM bonding site (14), to initiate the mechanosensing response, see Figure 7. There is a large literature on all aspects of this complex composition and assembly $(41,42)$, with a consensus emerging in the recent years that the dimer of $\alpha \beta$-integrins needs to be activated to expose the ECMbinding ligand. This activation occurs on binding the $\mathrm{N}$ terminal of talin $(33,43)$, while the C-terminal of talin is associated with paxillin, which in turn may associate with the focal adhesion targeting (FAT) domain (the C-terminal) of FAK. Both talin and paxillin also bind to cytoskeletal Factin; talin-actin links are known to be strengthened by vinculin $(44,45)$, but we see in the sequence of steps illustrated in Fig. 7 that there is no separate distinct step of complex assembly required to account for that.

It is worth noting that while in well-developed adhesions there is considerable actin-myosin contraction, it may well be that in early stage spreading, instead of grabbing onto an actin filament and being subject to a myosin pulling force, the nascent complex instead captures actin filaments undergoing retrograde flow. The captured actin flow could start to force the leading edge out, and help activate FAK. It seems likely that FAK activation is needed for the cell to properly recruit actin to push out the leading edge; FAK-null cells are much slower in the stage of early spreading (46).

If we then summarize the suggested four steps of the essential complex assembly, they are the following: [1] $\mathrm{N}$ terminal of talin binds and activates integrins; [2] integrins bind to ECM matrix, immobilizing the nascent complex, and C-terminal of talin binds to F-actin, thus forming the continuous force chain from cytoskeleton to ECM; [3] paxillin and/or vinculin bind to talin-actin assembly to complete the mechanical scaffold; [4] FAK binds to C-terminal of talin (and F-actin) by its FAT domain, and to the N-terminal of talin (and integrins) by its FERM domain. The sensor is now primed for action. The last step in the sequence is the opening of FAK domains, exposing the phosphorylation residues and the site for Src binding: the associate rate of FAK activation was calculated in (15). 
bioRxiv preprint doi: https://doi.org/10.1101/292367; this version posted April 4, 2018. The copyright holder for this preprint (which was not certified by peer review) is the author/funder. All rights reserved. No reuse allowed without permission.

The Supplementary Information gives more details of the classical kinetic theory (47) that predicts the early-time increase in the number of self-assembled nuclei of size $n$ to grow as a power-law law $\alpha t^{n-1}$, with the prefactor $\alpha$ proportional to the product of binding rates of each contributing protein. The reason for this power-law is that each individual assembly state has a linear time dependence (at early times), and the total rate is the product of them. This kinetic theory of protein aggregation is an example of a more general network theory (48) that shows how the kinetics of complex pathways is determined by their topology. The observed short-time behavior leads us to predict that there are four slow processes in the integrin-FAK mechanosensor assembly (thus giving $t^{4}$ growth), followed by the FAK activation process, completing the observed $t^{5}$ law. By 'slow', we mean any processes with rates of $k<1 \mathrm{~min}^{-1}$, because this was close to the resolution limit of our experiments; if a more advanced measurement of the kinetic curves such as in Fig. 5 could obtain data for the much shorter times after cell planting (say, within a second, or even sooner), this would resolve more fast assembly steps - and result in a steeper powerlaw in that very-short time region of the log-log plot. For instance, the dimerization of integrins, or the separate stages of talin binding and ECM binding, or other chaperon proteins attaching or assisting the complex construction could be resolved. But at our level of accuracy, the observed $\alpha t^{5}$ law leads to the 5-step sequence in Fig. 7.

Since the last step in our mechanosensing process has to be the FAK activation (the chemical signal generation), there are exactly four remaining kinetic steps left. That is, from the kinetic analysis we conclude that the sensor is an assembly of $n=5$ essential proteins (via four steps of binding, with the aggregate rate $k_{1} k_{2} k_{3} k_{4}$ ). We may not be certain of the order of assembly (e.g. talin-actin first, or paxillin-talin), and we may not be certain about which proteins are essential (although integrins, talin, FAK and F-actin are certain) - but we have to be sure that there are exactly four steps of the complex assembly. The product of binding rates in the prefactor $\alpha$ gives the overall Arrhenius exponential we saw in Fig. 6 with the total activation energy being the sum of all processes. It appears that the sum of binding energies of integrin-ECM, talin-integrin, talin-FAK, and FAK-actin is different in cells with different biological function: 3T3 fibroblasts have this sum $\left(70-\Delta G_{\mathrm{FAK}}\right) \approx 51 \mathrm{kcal} / \mathrm{mol}$, while EA endothelial cells have $\left(129-\Delta G_{\mathrm{FAK}}\right) \approx 110$ $\mathrm{kcal} / \mathrm{mol}$.

In this work, we have studied the population dynamics of spreading cells, and from this macroscopic observation we were able to infer details of the microscopic processes governing the overall cellular response to an external substrate. By linking the results to nucleation theory, we have found a novel way of looking at the onset of cell spreading as a problem of complex assembly. The next step will be to understand the development of mature structures once the cell spreading is well underway.

\section{Author contribution}

ALR carried out all experiments. SB, ALR and EMT carried out different elements of data analysis. SB and EMT wrote the paper.

\section{Acknowledgements}

The authors acknowledge many helpful discussions with $\mathrm{K}$. Franze, K. Chalut, A. B. Kolomeisky, and H. Welch. Critical comments of U. S. Schwarz, and experimental support in the cell culture lab by E. Nugent and F. Morgan are appreciated. This work has been funded by EPSRC (grants EP/M508007/1 and EP/J017639), and the Ernest Oppenheimer Trust in Cambridge.

\section{References}

1. Discher, D. E., P. Janmey, and Y.-L. Wang, 2005. Tissue cells feel and respond to the stiffness of their substrate. Science 310:1139-1143.

2. Yeung, T., P. C. Georges, L. A. Flanagan, B. Marg, M. Ortiz, M. Funaki, N. Zahir, W. Ming, V. Weaver, and P. A. Janmey, 2005. Effects of substrate stiffness on cell morphology, cytoskeletal structure, and adhesion. Cytoskeleton 60:24-34.

3. Engler, A. J., S. Sen, H. L. Sweeney, and D. E. Discher, 2006. Matrix elasticity directs stem cell lineage specification. Cell 126:677-689.

4. Hinz, B., 2007. Formation and function of the myofibroblast during tissue repair. J. Inv. Dermatol. 127:526-537.

5. Tomasek, J. J., G. Gabbiani, B. Hinz, C. Chaponnier, and R. A. Brown, 2002. Myofibroblasts and mechano-regulation of connective tissue remodelling. Nat. Rev. Mol. Cell Biol. 3:349-363.

6. Solon, J., I. Levental, K. Sengupta, P. C. Georges, and P. A. Janmey, 2007. Fibroblast adaptation and stiffness matching to soft elastic substrates. Biophys. J. 93:4453-4461.

7. Sinha, S., M. H. Hoofnagle, P. A. Kingston, M. E. McCanna, and G. K. Owens, 2004. Transforming growth factor- $\beta 1$ signaling contributes to development of smooth muscle cells from embryonic stem cells. Am. J. Physiol. Cell Physiol. 287:C1560-C1568.

8. Cheung, C., A. S. Bernardo, M. W. B. Trotter, R. A. Pedersen, and S. Sinha, 2012. Generation of human vascular smooth muscle subtypes provides insight into embryological origin-dependent disease susceptibility. Nat. Biotechnol. 30:165-173.

9. Alliston, T., L. Choy, P. Ducy, G. Karsenty, and R. Derynck, 2001. TGF- $\beta$-induced repression of CBFA 1 by $\mathrm{Smad} 3$ decreases cbfa1 and osteocalcin expression and inhibits osteoblast differentiation. EMBO J. 20:2254-2272.

10. Butcher, D. T., T. Alliston, and V. M. Weaver, 2009. A tense situation: forcing tumour progression. Nat. Rev. Cancer 9:108-122.

11. Schwarz, U. S., and S. A. Safran, 2013. Physics of adherent cells. Rev. Mod. Phys. 45:1327-1381.

12. Cockerill, M., M. K. Rigozzi, and E. M. Terentjev, 2015. Mechanosensitivity of the 2 nd kind: TGF- $\beta$ mechanism of cell

Biophysical Journal 00(00) 1-10 
sensing the substrate stiffness. PloS One 10:e0139959.

13. Mitra, S. K., D. A. Hanson, and D. D. Schlaepfer, 2005. Focal adhesion kinase: in command and control of cell motility. Nat. Rev. Mol. Cell Biol. 6:56-68.

14. Geiger, B., J. P. Spatz, and A. D. Bershadsky, 2009. Environmental sensing through focal adhesions. Nat. Rev. Mol. Cell Biol. 10:21-33.

15. Bell, S., and E. M. Terentjev, 2017. Focal adhesion kinase: the reversible molecular mechanosensor. Biophys. J. 112:24392450.

16. Ross, T. D., B. G. Coon, S. Yun, N. Baeyens, K. Tanaka, M. Ouyang, and M. A. Schwartz, 2013. Integrins in mechanotransduction. Curr. Opin. Cell Biol 25:613-618.

17. Huang, D. L., N. A. Bax, C. D. Buckley, W. I. Weis, and A. R. Dunn, 2017. Vinculin forms a directionally asymmetric catch bond with F-actin. Science 357:703-706.

18. Buckley, C. D., J. Tan, K. L. Anderson, D. Hanein, N. Volkmann, W. I. Weis, W. J. Nelson, and A. R. Dunn, 2014. The minimal cadherin-catenin complex binds to actin filaments under force. Science 346:1254211.

19. Cuvelier, D., M. Théry, Y.-S. Chu, S. Dufour, J.-P. Thiéry, M. Bornens, P. Nassoy, and L. Mahadevan, 2007. The universal dynamics of cell spreading. Curr. Biol. 17:694-699.

20. Brill-Karniely, Y., N. Nisenholz, K. Rajendran, Q. Dang, R. Krishnan, and A. Zemel, 2014. Dynamics of cell area and force during spreading. Biophys. J. 107:L37-L40.

21. Li, J., D. Han, and Y.-P. Zhao, 2013. Kinetic behaviour of the cells touching substrate: the interfacial stiffness guides cell spreadin. Sci. Rep. 4:3910.

22. Döbereiner, H.-G., B. Dubin-Thaler, G. Giannone, H. S. Xenias, and M. P. Sheetz, 2004. Dynamic phase transitions in cell spreading. Phys. Rev. Lett. 93:108105.

23. Xiong, Y., P. Rangamani, M.-A. Fardin, A. Lipshtat, B. DubinThaler, O. Rossier, M. P. Sheetz, and I. R., 2010. Mechanisms controlling cell size and shape during isotropic cell spreading. Biophys. J. 98:2136-2146.

24. Bouïs, D., G. A. P. Hospers, C. Meijer, G. Molema, and N. H. Mulder, 2001. Endothelium in vitro: a review of human vascular endothelial cell lines for blood vessel-related research. Angiogenesis 4:91-102.

25. Freshney, R. I., 2010. Culture of Animal Cells. John Wiley \& Sons, Inc.

26. Todaro, G. J., and H. Green, 1963. Quantitative studies of the growth of mouse embryo cells in culture and their development into established lines. J. Cell Biol. 17:299-313.

27. Rocha, A., M. Hahn, and H. Liang, 2010. Critical fluid shear stress analysis for cell-polymer adhesion. J. Mater. Sci. 45:811-817.

28. Edgell, C.-J., C. C. McDonald, and J. B. Graham, 1983. Permanent cell line expressing human factor VIII-related antigen established by hybridization. Proc. Natl. Acad. Sci. USA 80:3734-3737.

29. Han, Y.-L., Q. Xu, Z. Lu, and J.-Y. Wang, 2013. Cell adhesion on zein films under shear stress field. Coll. Surf. B: Biointerfaces 111:479-485.

30. Kuhlmann, I., 1995. The prophylactic use of antibiotics in cell culture. Cytotechnology 19:95-105.

31. Cruickshank, C. N. D., and E. J. L. Lowbury, 1952. Effect of antibiotics on tissue cultures of human skin. Brit. Med. J.
2:1070-1072.

32. Dubin-Thaler, B. J., G. Giannone, H.-G. Döbereiner, and M. P. Sheetz, 2004. Nanometer analysis of cell spreading on matrixcoated surfaces reveals two distinct cell states and STEPs. Biophys. J. 86:1794-1806.

33. Margadant, F., L. L. Chew, X. Hu, H. Yu, N. Bate, X. Zhang, and M. Sheetz, 2011. Mechanotransduction in vivo by repeated talin stretch-relaxation events depends upon vinculin. PLoS Biol. 9:e1001223.

34. Zhou, J., C. Aponte-Santamaría, S. Sturm, J. T. Bullerjahn, A. Bronowska, and F. Gräter, 2015. Mechanism of focal adhesion kinase mechanosensing. PLoS Comp. Biol. 11:e1004593.

35. Huveneers, S., and E. H. J. Danen, 2009. Adhesion signalingcrosstalk between integrins, Src and Rho. J. Cell Sci. 122:1059-1069.

36. Schwartz, M. A., and S. J. Shattil, 2000. Signaling networks linking integrins and Rho family GTPases. Trends Biochem. Sci. 25:388-391.

37. Pajic, M., D. Herrmann, C. Vennin, J. R. W. Conway, V. T. Chin, A.-K. E. Johnsson, H. C. E. Welch, and P. Timpson, 2015. The dynamics of Rho GTPase signaling and implications for targeting cancer and the tumor microenvironment. Small GTPases 6:123-133.

38. Zaidel-Bar, R., S. Itzkovitz, A. Ma'ayan, R. Iyengar, and B. Geiger, 2007. Functional atlas of the integrin adhesome. Nat. Cell Biol. 9:858-867.

39. Sieg, D. J., C. R. Hauck, D. Ilic, C. K. Klingbeil, E. Schaefer, C. H. Damsky, and D. D. Schlaepfer, 2000. FAK integrates growth-factor and integrin signals to promote cell migration. Nat. Cell Biol. 2:249-256.

40. Calderwood, D. A., and M. H. Ginsberg, 2003. Talin forges the links between integrins and actin. Nat. Cell Biol. 5:694-697.

41. Parsons, J. T., 2003. Focal adhesion kinase: the first ten years. J. Cell Sci. 116:1409-1416.

42. Giancotti, F. G., 2000. Complexity and specificity of integrin signalling. Nat. Cell Biol. 2:E13-E14.

43. Hytönen, V. P., and B. Wehrle-Haller, 2016. Mechanosensing in cell-matrix adhesions-Converting tension into chemical signals. Exp. Cell Res. 343:35-41.

44. Hemmings, L., D. J. Rees, V. Ohanian, S. J. Bolton, A. P. Gilmore, B. Patel, H. Priddle, J. E. Trevithick, R. O. Hynes, and D. R. Critchley, 1996. Talin contains three actin-binding sites each of which is adjacent to a vinculin-binding site. J. Cell Sci. 109:2715-2726.

45. Yao, M., B. T. Goult, H. Chen, P. Cong, M. P. Sheetz, and J. Yan, 2014. Mechanical activation of vinculin binding to talin locks talin in an unfolded conformation. Sci. Rep. 4:4610.

46. Owen, J. D., P. J. Ruest, D. W. Fry, and S. K. Hanks, 1999. Induced focal adhesion kinase (FAK) expression in FAK-null cells enhances cell spreading and migration requiring both auto-and activation loop phosphorylation sites and inhibits adhesion-dependent tyrosine phosphorylation of Pyk2. Mol. Cell Biol. 19:4806-4818.

47. Hofrichter, J., P. D. Ross, and W. A. Eaton, 1974. Kinetics and mechanism of deoxyhemoglobin S gelation: a new approach to understanding sickle cell disease. Proc. Natl. Acad. Sci. USA 71:4864-4868.

48. Valleriani, A., X. Li, and A. B. Kolomeisky, 2014. Unveiling the hidden structure of complex stochastic biochemical 
bioRxiv preprint doi: https://doi.org/10.1101/292367; this version posted April 4, 2018. The copyright holder for this preprint (which was not certified by peer review) is the author/funder. All rights reserved. No reuse allowed without permission.

networks. J. Chem. Phys. 140:064101. 